\title{
The 3D Modeling System for Bioaerosol Distribution Based on Planar Laser-Induced Fluorescence
}

\author{
Siying Chen ${ }^{1}$, Yuanyuan Chen ${ }^{1}$, Yinchao Zhang ${ }^{1}$, Pan Guo ${ }^{1}$, He Chen ${ }^{1, *}$ and Huiyun $\mathrm{Wu}^{2, *}$ \\ 1 School of Optics and Photonics, Beijing Institute of Technology, Beijing 100081, China; csy@bit.edu.cn (S.C.); \\ 3120160297@bit.edu.cn (Y.C.); ychang@bit.edu.cn (Y.Z.); guopan@bit.edu.cn (P.G.) \\ 2 Academy of Military Medical Sciences, Academy of Military Sciences, Beijing 100850, China \\ * Correspondence: shinianshao@bit.edu.cn (H.C.); Huiyunwu740@126.com (H.W.)
}

check for updates

Citation: Chen, S.; Chen, Y.; Zhang, Y.; Guo, P.; Chen, H.; Wu, H. The 3D Modeling System for Bioaerosol Distribution Based on Planar Laser-Induced Fluorescence. Sensors 2021, 21, 2607. https://doi.org/ $10.3390 / \mathrm{s} 21082607$

Academic Editor: Carmine Serio

Received: 16 March 2021

Accepted: 31 March 2021

Published: 8 April 2021

Publisher's Note: MDPI stays neutral with regard to jurisdictional claims in published maps and institutional affiliations.

Copyright: (C) 2021 by the authors. Licensee MDPI, Basel, Switzerland. This article is an open access article distributed under the terms and conditions of the Creative Commons Attribution (CC BY) license (https:/ / creativecommons.org/licenses/by/ $4.0 /)$.

\begin{abstract}
Although it is quite challenging to image and analyze the spatial distribution of bioaerosols in a confined space, a three-dimensional (3D) modeling system based on the planar laser-induced fluorescence (PLIF) technique is proposed in this paper, which is designed to analyze the temporal and spatial variations of bioaerosol particles in a confined chamber. The system employs a continuous planar laser source to excite the fluoresce, and a scientific complementary metal oxide semiconductor (sCMOS) camera to capture images of $2048 \times 2048$ pixels at a frame rate of $12 \mathrm{~Hz}$. While a sliding platform is moving back and forth on the track, a set of images are captured at different positions for 3D reconstruction. In this system, the $3 \mathrm{D}$ reconstruction is limited to a maximum measurement volume of about $50 \mathrm{~cm} \times 29.7 \mathrm{~cm} \times 42 \mathrm{~cm}$, with a spatial resolution of about $0.58 \mathrm{~mm} \times 0.82 \mathrm{~mm} \times 8.33 \mathrm{~mm}$, and a temporal resolution of $5 \mathrm{~s}$. Experiments were carried out to detect the PLIF signals from fluorescein aerosols in the chamber, and then $3 \mathrm{D}$ reconstruction was used to visualize and analyze the diffusion of aerosol particles. The results prove that the system can be applied to clearly reconstruct the $3 \mathrm{D}$ distribution and record the diffusion process of aerosol particles in a confined space.
\end{abstract}

Keywords: bioaerosol; PLIF; concentration; 3D; reconstruction

\section{Introduction}

Bioaerosols usually refer to the aerosols containing biological particles such as bacteria, viruses, and pollen, which are strongly associated with human lives [1,2]. The leakage and spread of infectious or allergenic biological aerosols may significantly infect the human respiratory system, and even cause nerve damage [3]. Now, increasing attention has been directed toward the effects of indoor fungal bioaerosol exposure on health and safety, especially in an enclosed space [4]. Generally, the factors, such as airflows and walking activities of indoor occupants, may affect the variation of fungal and bacterial bioaerosols in indoor environments [5]. Therefore, it is of great significance to measure and analyze the distribution of biological aerosols in a confined environment. The laser-induced fluorescence (LIF) is a highly sensitive technique that can discriminate between different biological particles and non-biological particles and has been applied for effective detection and characterization of bioaerosols [6-8].

The LIF-based standoff detection on bioaerosols has been widely applied [9-11]. Based on the fluorescence images of different spectral bands, the LIF system can identify bacteria contamination on the target object [12]. Meanwhile, several approaches have been introduced to optimize the three-dimensional (3D) imaging system based on planar LIF (PLIF) and volumetric LIF (VLIF) techniques. For example, Cho et al. developed a scanned PLIF system to detect the relative concentration of $\mathrm{OH}$ in multiphase combustion flow fields [13]. By scanning the laser sheet across different spatial locations, multiple images for 3D imaging can be effectively captured with the PLIF technique. Miller et al. presented a 3D PLIF imaging system using toluene as the tracer and visualized the co-flow jet mixing with ambient air [14]. Instead of scanning the laser sheet, the VLIF system can 
capture the volumetric fluorescence from different angles using multiple cameras, with a higher temporal and spatial resolution $[15,16]$. The VLIF technique has also been applied to image 3D concentration fields in the turbulent gaseous free jet using four complementary metal oxide semiconductor (CMOS) cameras [17]. Furthermore, Li et al. have studied the reconstruction of 3D flame structures using VLIF signals from eight camera views [18].

Conventional LIF technique generally detects biological particles along one line or at a specific position in the measurement environment [9-12,19]. Accordingly, only limited details could be captured from fluorescence signals for analyzing the distribution of biological aerosols. With a high temporal and spatial resolution, the 3D imaging system based on PLIF or VLIF techniques can be used to analyze the spatial distribution and dynamic process of the target in a non-intrusive manner. This system has been applied extensively for studies in combustion diagnosis, jet flows, and catalytic reactions [20-22]. However, the 3D LIF measurement generally requires the scanned laser sheet or multiple cameras [23,24], which is optically complex and less flexible. Additionally, the measurement volume of a $3 \mathrm{D}$ imaging system is limited and normally does not exceed $50 \mathrm{~mm} \times 50 \mathrm{~mm} \times 50 \mathrm{~mm}$. Therefore, few studies have been reported on applying the PLIF or VIF techniques for 3D imaging of bioaerosols in a larger space.

In this study, we designed and built a 3D modeling system to capture images of fluorescence intensity and visualize the spatial distribution of bioaerosols using the PLIF technique. Different from the multi-camera detection system, this system applies a planar laser beam from the continuous-wave $(\mathrm{CW})$ laser to excite the target particles, and a scientific CMOS (sCMOS) camera to capture fluorescence images. The methods of image denoising, geometric correction, and $3 \mathrm{D}$ reconstruction are employed to reconstruct the $3 \mathrm{D}$ distribution of the target particles in a $500 \mathrm{~mm} \times 500 \mathrm{~mm} \times 1000 \mathrm{~mm}$ chamber. It was found that a new approach can be applied to achieve 3D imaging of fluorescein aerosols with sufficient temporal and spatial resolution in a larger volume. Therefore, the paper presents and discusses the feasibility of using the PLIF technique to achieve 3D imaging of the relative concentration of bioaerosols in the enclosed environment.

\section{System and Experiment}

\subsection{Structure and Equipment}

As shown in Figure 1, the schematic of the 3D modeling system is presented. With a wavelength of $450 \mathrm{~nm}$ and laser power of $100 \mathrm{~mW}$, a beam-shaping device is equipped on the CW laser to form a laser sheet at an angle of $60^{\circ}$. The detection plane forms an angle of $45^{\circ}$ with the $\mathrm{Y}-\mathrm{Z}$ plane. The sCMOS camera (HAMAMATSU ORCA-Flash4.0 V3, Hamamatsu, Japan) with an optical lens (Kowa LM16XC) is employed to capture PLIF images of $2048 \times 2048$ pixels, with each pixel size of $6.5 \mu \mathrm{m} \times 6.5 \mu \mathrm{m}$. The aerosol chamber is made of quartz glass, with a volume of $500 \mathrm{~mm} \times 500 \mathrm{~mm} \times 1000 \mathrm{~mm}$. The bandpass filter (Edmund \#33-331, Barrington, New Jersey, USA) is placed in front of the lens to select the specific fluorescence signals in the wavelength range from $500 \mathrm{~nm}$ to $600 \mathrm{~nm}$. The laser and camera are fixed on a sliding platform, with the imaging orientation perpendicular to the laser plane. Driven by a motor, the sliding platform can move back and forth along the Z-axis. The main parameters of the 3D modeling system are listed in Table 1.

Table 1. The main parameters of the system.

\begin{tabular}{ccc}
\hline Component & Parameter & Value \\
\hline \multirow{2}{*}{ Laser } & Laser Power & $100 \mathrm{~mW}$ \\
& Laser Wavelength & $450 \mathrm{~nm}$ \\
Lens & Focal Length & $16 \mathrm{~mm}$ \\
& Imaging Distance & $30 \mathrm{~cm}$ \\
Camera & Quantum Efficiency & $0.82(500-600 \mathrm{~nm})$ \\
& Cell Size & $6.5 \mu \mathrm{m} \times 6.5 \mu \mathrm{m}$ \\
\hline
\end{tabular}




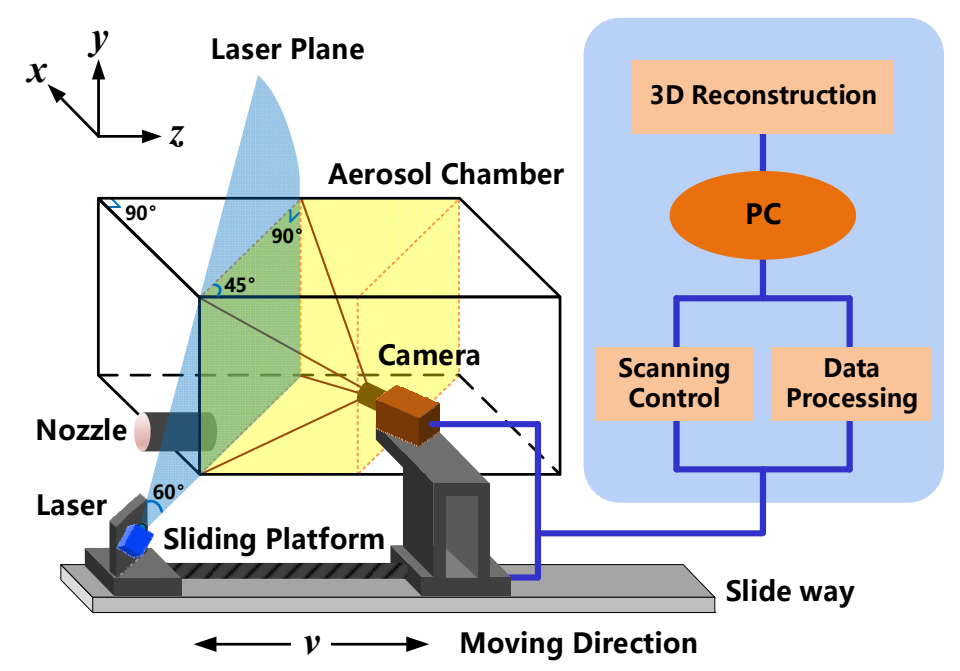

Figure 1. Schematic of the 3D modeling system.

\subsection{Experimental Design}

With a high quantum yield, the fluorescein $\left(\mathrm{C}_{20} \mathrm{H}_{12} \mathrm{O}_{5}\right)$ was employed as the reagent for fluorescence excitation in the experiments. The yielded fluorescence signals peaked at the wavelength of approximately $510 \mathrm{~nm}$, with the excitation laser of $450 \mathrm{~nm}$ [25].

In the experiments, fluorescein solution was atomized by a nebulizer at an atomization rate of $0.5 \mathrm{~mL} / \mathrm{s}$ to generate test aerosols with the mass median diameter of $3.9 \mu \mathrm{m}$, and aerosol particles with particle size smaller than $5 \mu \mathrm{m}$ exceed $65 \%$. The resulting particles were filled into the aerosol chamber through a nozzle. When the laser excited the fluorescein, PLIF images were captured by the camera with the frame rate of $12 \mathrm{~Hz}$. The indoor lights were switched off during the experiments.

The following experiments were carried out to validate the system functions. In the first step, when the fluorescein solution at a concentration of $0.1 \mathrm{~g} / \mathrm{L}$ was atomized into the chamber, the two-dimensional (2D) PLIF images were captured by the camera and went through the image denoising, geometric correction, and coordinate transformation. Then, the fluorescein solutions at concentrations of $0.05 \mathrm{~g} / \mathrm{L}$ and $0.1 \mathrm{~g} / \mathrm{L}$ were atomized into the chamber separately, 2D PLIF images were continuously collected at the same position to compare the changes of the 2D distribution of fluorescein solution aerosols at different concentrations. Finally, we atomized $0.1 \mathrm{~g} / \mathrm{L}$ fluorescein solution, and collected PLIF images at different positions along the Z-axis for $30 \mathrm{~s}$, while the sliding platform was moving at a speed of $10 \mathrm{~cm} / \mathrm{s}$. And the captured images were used to study the 3D reconstruction of relative concentrations of aerosols, and the $3 \mathrm{D}$ variation process of the aerosols.

\section{Data Processing}

\subsection{Image Denoising}

In order to increase the signal-to-noise ratio (SNR) and reduce the stray light during the process of collecting PLIF images, the background subtraction and pixel binning methods were applied for image denoising. The original PLIF images captured at the resolution of $2048 \times 2048$ pixels were resized to $512 \times 512$ pixels by means of a $4 \times 4$ pixel binning process. The imaging plane covered an area of $42 \mathrm{~cm} \times 42 \mathrm{~cm}$ in the camera. Therefore, the resolution of the 2D image was about $0.82 \mathrm{~mm} \times 0.82 \mathrm{~mm}$. Before aerosols entered the chamber, the background noises were collected when the laser was switched on. Background subtraction was performed by subtracting the noise image from the original 
PLIF images. Additionally, the wavelet thresholding technique was applied for image denoising. The soft threshold function can be written as [26]:

$$
w_{\delta}=\left\{\begin{array}{cc}
\operatorname{sgn}(w)(|w|-\delta), & |w| \geq \delta \\
0, & |w|<\delta
\end{array}\right.
$$

where $w$ is the coefficient vector by wavelet decomposition of the image, $\delta$ is the threshold, which can be calculated as [27]:

$$
\delta=\left\{\begin{array}{cc}
\sigma\left[0.3936+0.1829\left(\frac{\ln N}{\ln 2}\right)\right], & N>32 \\
0, & N \leq 32
\end{array}\right.
$$

where $N$ is the length of the signal vector, $\sigma$ represents the variance of noise, which can be written as [27]:

$$
\sigma=\text { median }\left|f_{i}\right| / 0.6745
$$

where $f_{i}$ is the wavelet coefficient vector at unit scale.

\subsection{Geometric Correction of Laser Intensity}

As shown in Figure 2, the laser source is a $60^{\circ}$ fan-shaped beam, the laser energy in each pixel of the detection plane is different and should be corrected. Without considering the influence of aerosol particles, the geometric correction of the laser intensity is discussed and analyzed below.

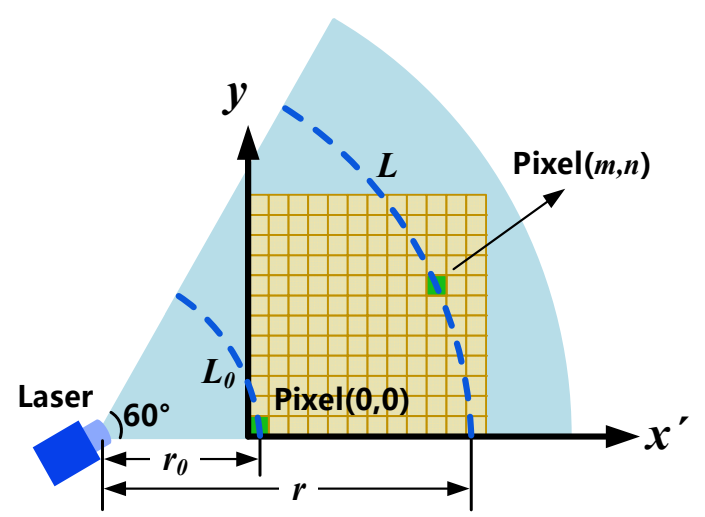

Figure 2. Schematic of laser detection plane.

$r$ is defined as the detection distance of the pixel $(m, n), L$ is defined as the arc length of the laser plane with the detection distance of $r . r_{0}$ is the detection distance of pixel $(0,0)$, $L_{0}$ is defined as the arc length with the detection distance of $r_{0}$. The ratio of laser intensity $I_{0}$ at the location of the pixel $(0,0)$ and laser intensity $I(m, n)$ can be expressed as:

$$
\frac{I_{0}}{I(m, n)}=\frac{L(m, n)}{L_{0}}=\frac{r(m, n)}{r_{0}}=\frac{\sqrt{\left(r_{0}+m\right)^{2}+n^{2}}}{r_{0}}
$$

Thus, the geometric correction ratio is described as:

$$
P(m, n)=\frac{I_{0}}{I(m, n)}=\frac{r_{0}}{\sqrt{\left(r_{0}+m\right)^{2}+n^{2}}}
$$

As shown in Figure 3, the ratio of laser intensity at each pixel is related to the detection distance. It is obvious that the laser intensity gradually gets weakened from the lower-left corner to the upper right corner. The uneven distribution of laser intensity imposes a 
significant effect on PLIF signals. Therefore, each pixel intensity of PLIF images should be a multiple of the geometric correction ratio to get the accurate fluorescence signal.

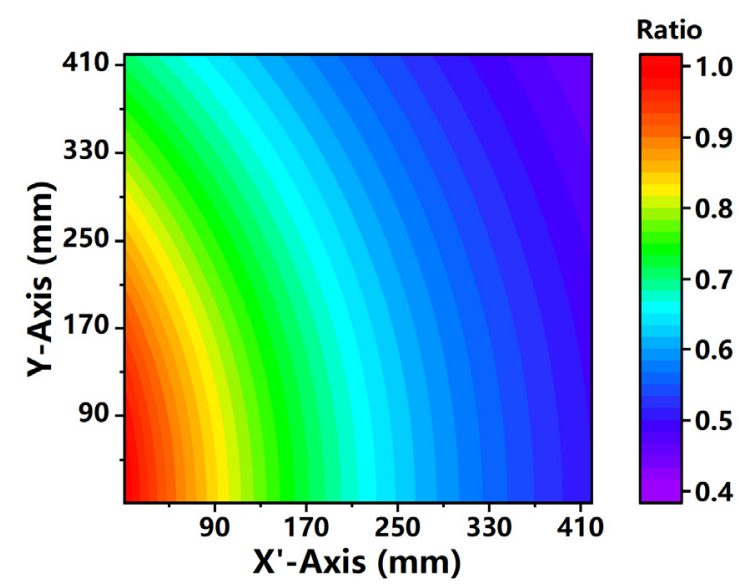

Figure 3. The ratios of laser intensities (the imaging plane is $42 \mathrm{~cm} \times 42 \mathrm{~cm}$ ).

\subsection{D Reconstruction}

The pixels of PLIF images are identified by 3D spatial coordinates and the position of the pixel $(0,0, p)$ in each PLIF image is recorded according to the position of the sliding platform. As shown in Figure 4, the original 3D spatial coordinates of each pixel are recorded as $(m, n, p)$ in image A. However, the laser plane forms an angle of $45^{\circ}$ with the chamber sidewall. In order to demonstrate the right $3 \mathrm{D}$ reconstruction, the spatial position of the pixel $(m, n, p)$ should be corrected as $\left(m^{\prime}, n^{\prime}, p^{\prime}\right)$ in image B.

$$
\begin{gathered}
m^{\prime}=\frac{m}{\sqrt{2}} \\
n^{\prime}=n \\
p^{\prime}=p+\frac{m}{\sqrt{2}}
\end{gathered}
$$
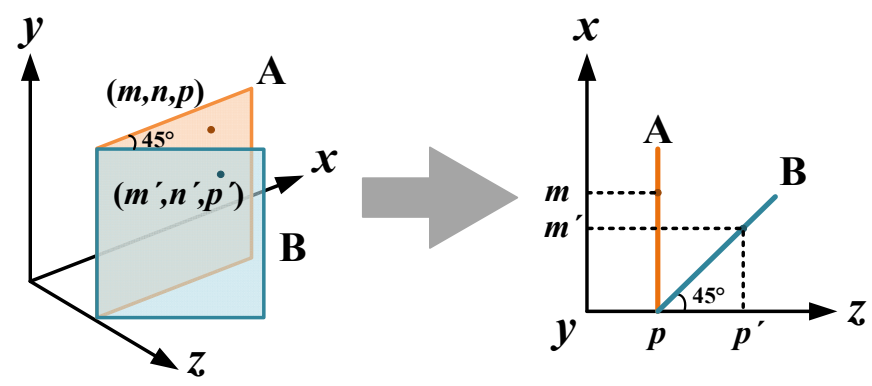

Figure 4. Schematic of coordinate transformation.Therefore, a fundamental step in the 3D reconstruction is to realize the spatial coordinate transformation from image A to image $\mathrm{B}$.

\section{Result and Discussion}

\subsection{Analysis of Fluorescence Attenuation}

As shown in Figure 5, the light is emitted from the laser source to the target point $S$, and then the fluorescence light excited from the point $S$ enters the sCMOS camera. Both laser light and fluorescence light will be attenuated along the path by the aerosols in the chamber. We define $S$ as the intensity of the excited fluorescence, without considering 
aerosol attenuation. Therefore, the ratio of detected fluorescence intensity $S_{d}$ to $S$ can be approximately expressed as [28]:

$$
\frac{S_{d}}{S}=10^{-\left(A_{e x}+A_{e m}\right) / 2}
$$

where $A_{e x}$ is the absorbance at the excitation wavelength, $A_{e m}$ represents the absorbance of fluorescence.

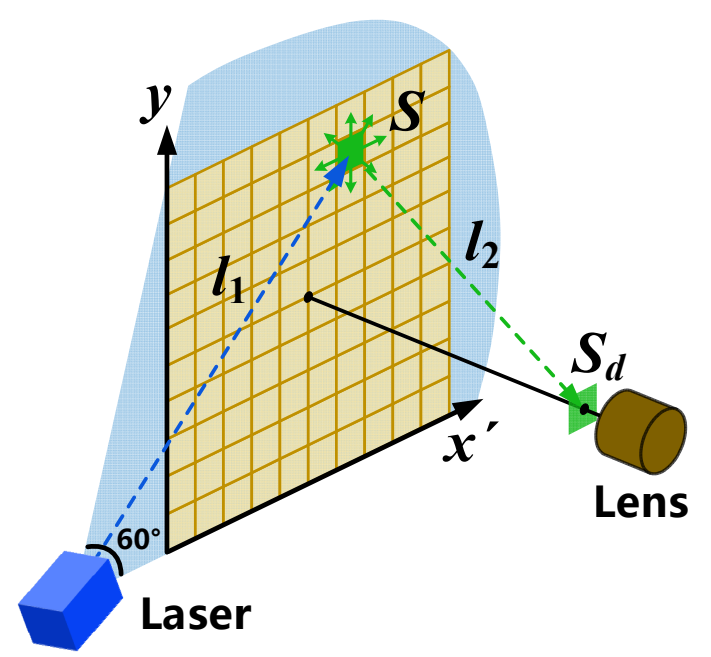

Figure 5. Schematic of fluorescence attenuation.

According to Lambert-Beer's law, the absorbance can be obtained as below [29]:

$$
A_{e x}+A_{e m}=\varepsilon_{1} \int_{0}^{l_{1}} C_{1}(l) d l+\varepsilon_{2} \int_{0}^{l_{2}} C_{2}(l) d l
$$

where $\varepsilon_{1}$ and $\varepsilon_{2}$ are the extinction coefficients for the excitation and emission wavelengths respectively, $l_{1}$ is the optical path of laser light, $l_{2}$ is the optical path of fluorescence, $C_{1}(l)$ and $C_{2}(l)$ are the local concentrations of aerosols.

According to Equation (8) and Figure 5, the detected fluorescence intensity $S_{d}$ at a specific pixel depends on the concentration distribution of fluorescein in the chamber, the position of the conjugated object point, and extinction coefficients $\left(\varepsilon_{1}, \varepsilon_{2}\right)$ which are considered as constants with negligible changes. In the experiments, it was observed that the fluorescein solution samples exhibited a maximum concentration of $0.1 \mathrm{~g} / \mathrm{L}$, with an atomization rate of $0.5 \mathrm{~mL} / \mathrm{s}$. As mentioned above, the aerosol chamber has a volume of $250 \mathrm{~L}$, and the atomization process took place within $50 \mathrm{~s}$, so the average concentration peaked at $0.01 \mathrm{mg} / \mathrm{L}$. Because the fluorescein characterized the non-uniform concentration distribution in the chamber, we assumed that the concentration at a certain location is dozens of tens times higher than the average value. When the concentration was 30, 40, and 50 times higher than the average value, the ratio $\left(S_{d} / S\right)$ was calculated according to Equation (7), with the results shown in Figure 6. It was found that the ratio decreased with the increasing detection distance, showing a downward trend. The light attenuation rate at a certain concentration could be obtained by subtracting the ratio from 1 , which was $2.87 \%(0.3 \mathrm{mg} / \mathrm{L}), 3.80 \%(0.4 \mathrm{mg} / \mathrm{L})$ and $4.73 \%(0.5 \mathrm{mg} / \mathrm{L})$, respectively. It proves that the light attenuation due to aerosols did not exceed $5 \%$ even when the concentration reached $0.5 \mathrm{mg} / \mathrm{L}$ which was 50 times higher than the average value. Therefore, we analyzed the distribution of relative concentrations based on the detected fluorescence intensity in the following data processing, without considering the light attenuation. 


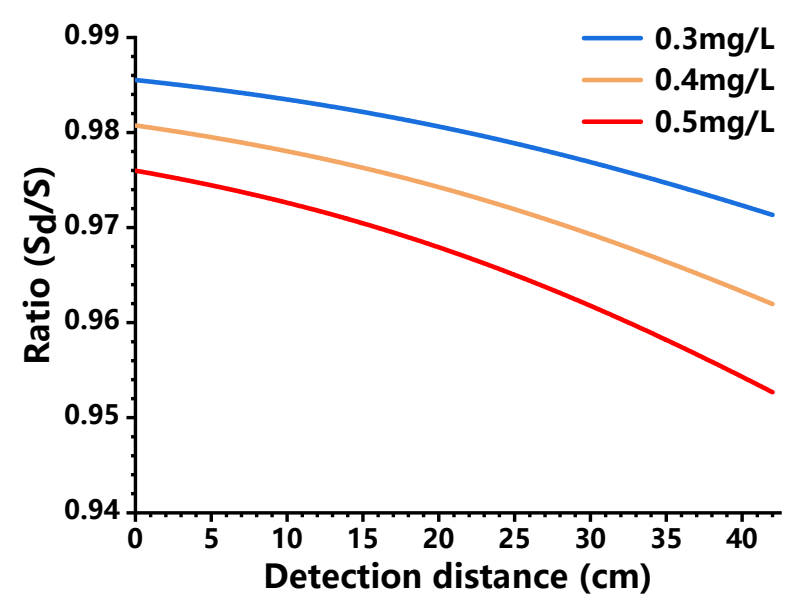

Figure 6. Ratio as a function of detection distance at three different concentrations $(0.3 \mathrm{mg} / \mathrm{L}$, $0.4 \mathrm{mg} / \mathrm{L}, 0.5 \mathrm{mg} / \mathrm{L})$.

\subsection{Analysis of Image Processing}

To collect the PLIF images, we prepared a fluorescein solution at the concentration of $0.1 \mathrm{~g} / \mathrm{L}$, which was atomized by a nebulizer into the chamber with an atomization rate of $0.5 \mathrm{~mL} / \mathrm{s}$. The imaging system continuously captured the PLIF images in the atomization process. Figure 7 shows the PLIF images captured at the 20th second in different preprocessing stages. As shown in Figure 7a, it's hard to distinguish the intensity distribution through PLIF images processed with background subtraction and pixel binning methods. However, the smoothed PLIF images which went through background subtraction, pixel merging, and wavelet denoising, present a more clear and virtualized intensity distribution, as shown in Figure $7 \mathrm{~b}$. In addition to the image processing methods used in Figure $7 \mathrm{a}, \mathrm{b}$, a geometric correction was applied to analyze the laser intensity, and it's found that the fluorescence intensity of images is significantly improved and the difference of PLIF signal intensities is more obvious, as shown in Figure 7c. Therefore, according to the comparative results, the distribution and diffusion of particles can be further recorded and analyzed.

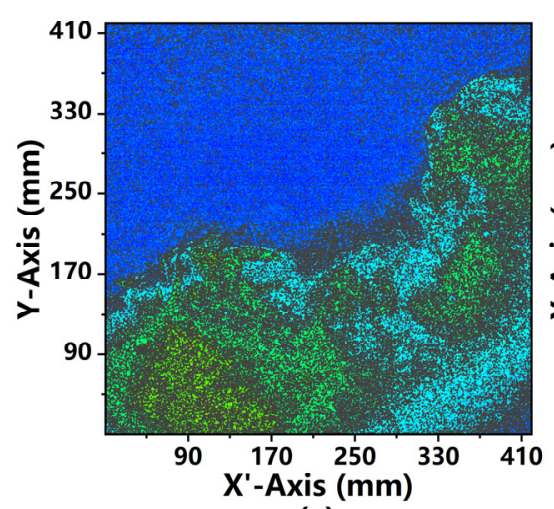

(a)

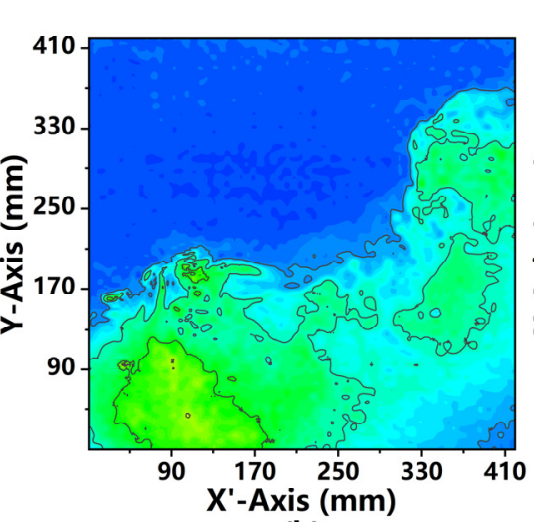

(b)

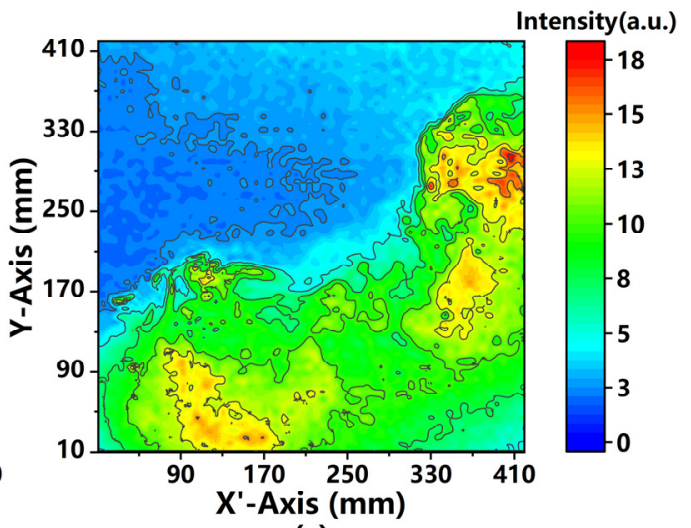

(c)

Figure 7. The 2D intensity distribution of planar laser-induced fluorescence (PLIF) images captured at the 20th second. The signals in (a) were processed with background subtraction and pixel merging, the signals in (b) were processed with background subtraction, pixel merging, and wavelet denoising, and the signals in (c) were further processed with geometric correction, in addition to the above mentioned three methods. The concentration of the atomized fluorescein solution was $0.1 \mathrm{~g} / \mathrm{L}$, and the signals were obtained with the laser power of $100 \mathrm{~mW}$, a $25 \mathrm{~ms}$ frame, and an atomization rate of $0.5 \mathrm{~mL} / \mathrm{s}$. 


\subsection{Comparison of $2 D$ Intensity Distributions}

To assess the system performance of detecting the 2D distributions of aerosols, we selected the fluorescein solutions at two different concentrations for comparison. In the experiments, fluorescein solutions were atomized by a nebulizer with an atomization rate of $0.5 \mathrm{~mL} / \mathrm{s}$. As shown in Figure 8, the PLIF images at the 2.5th, 7.5th, and 12.5th seconds were collected while the sliding platform remained stationary. The fluorescein solutions at a concentration of $0.05 \mathrm{~g} / \mathrm{L}$ were prepared for atomization in Figure 8a1-a3, and $0.1 \mathrm{~g} / \mathrm{L}$ solutions were prepared in Figure 8b1-b3.

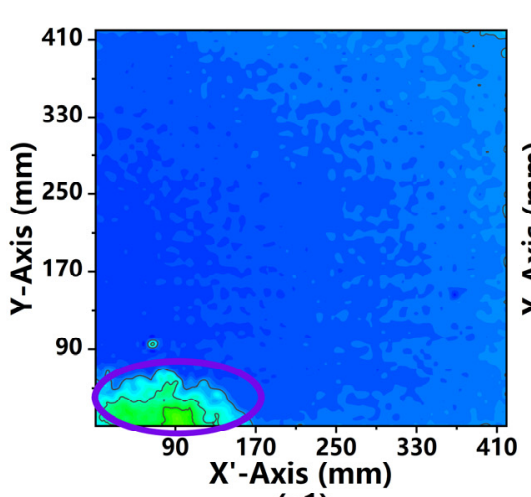

(a1)

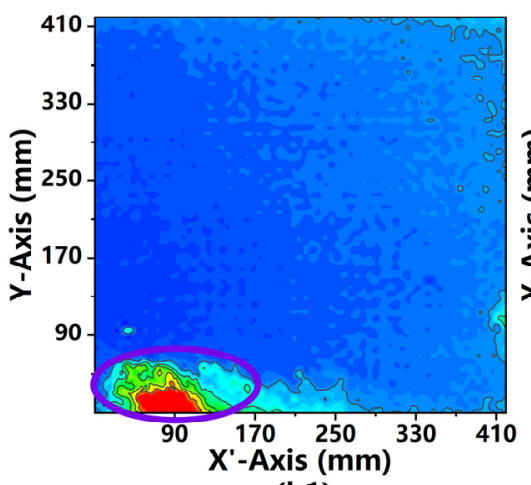

(b1)

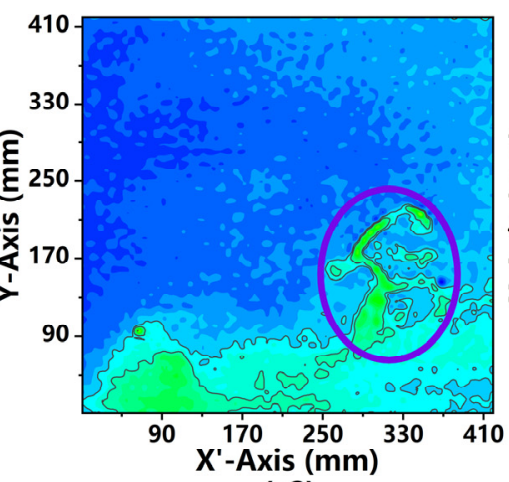

(a2)

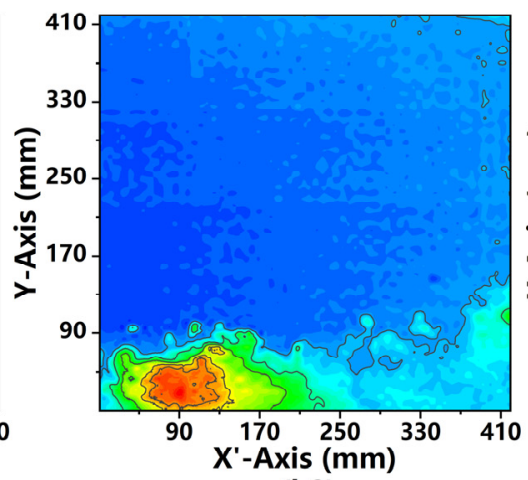

(b2)

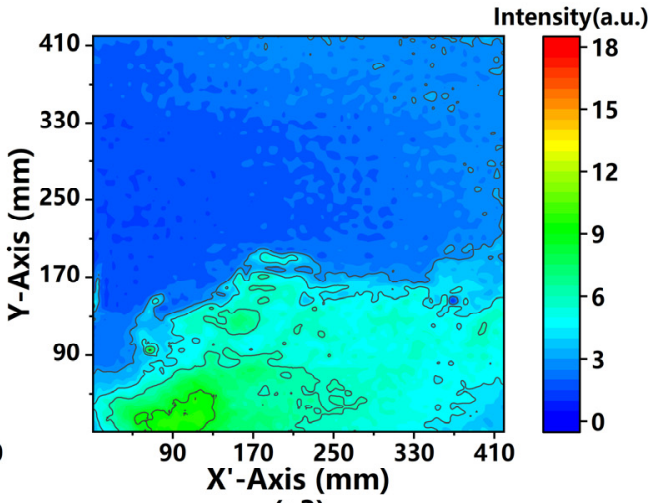

(a3)

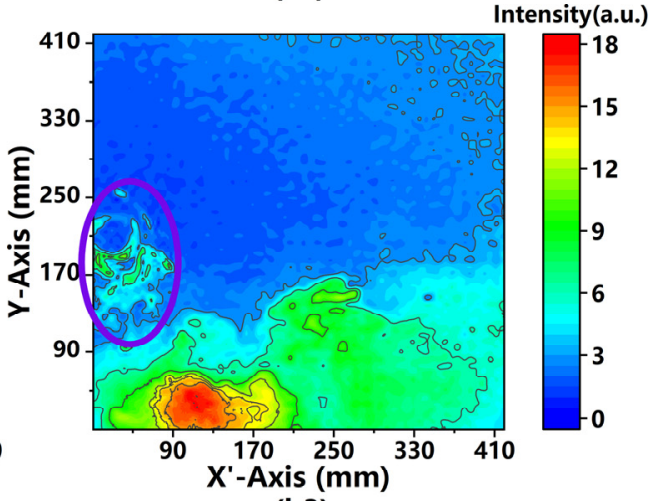

(b3)

Figure 8. The 2D intensity distribution of PLIF images captured at the 2.5th, 7.5 th, and 12.5 th second. The concentration of the atomized fluorescein solution in (a1), (a2) and (a3) is $0.05 \mathrm{~g} / \mathrm{L}$, and the concentration of the atomized fluorescein solution in (b1), (b2) and (b3) is $0.1 \mathrm{~g} / \mathrm{L}$. The signals were obtained with the laser power of $100 \mathrm{~mW}$, a $25 \mathrm{~ms}$ frame, and an atomization rate of $0.5 \mathrm{~mL} / \mathrm{s}$.

As outlined in purple in Figure 8a1,b1, it can be observed that PLIF signals present a high intensity at the 2.5 th second. This is because the nozzle was placed in the lower part of the chamber. PLIF signals were getting stronger with the injection of particles. Figure $8 \mathrm{a} 2, \mathrm{~b} 2$ exhibit the $2 \mathrm{D}$ distribution of particles at the 7.5 th second. A significant increase in signals can be observed at the bottom of the entire cross-sectional area, indicating that the fluorescein aerosols first diffused across the lower part of the chamber. In addition, as outlined in purple in Figure 8a2,b3, the unusual distribution of PLIF signals can be observed, which might result from small-scale turbulence. Figure 8a3,b3 shows that the 2D distribution of upward-diffused particles at the 12.5th second. In addition, the peak intensity of signals is nearly twice as much in Figure 8b1-b3 as in Figure 8a1-a3. According to the PLIF images, the 2D distribution of fluorescein solution particles can clearly reflect the bottom-up diffusion. 


\subsection{Diffusion Process of 3D Distribution}

To evaluate the system performance of monitoring 3D distribution and diffusion of aerosols, we collected PLIF images at different positions along the Z-axis during the atomization process. The $0.1 \mathrm{~g} / \mathrm{L}$ fluorescein solution was atomized by a nebulizer with an atomization rate of $0.5 \mathrm{~mL} / \mathrm{s}$ in the experiment. While the sliding platform moved back and forth with a speed of $10 \mathrm{~cm} / \mathrm{s}$, the camera captured the PLIF images with an exposure time of $25 \mathrm{~ms} /$ frame and a frame rate of $12 \mathrm{~Hz}$. The laser plane forms an angle of $45^{\circ}$ with the chamber sidewall. Through coordinates transformation of image pixels, the 3D distributions of fluorescein solution particles were reconstructed. The 60 PLIF images were taken to reconstruct a 3D distribution at a temporal resolution of $5 \mathrm{~s}$ and a spatial resolution of about $0.58 \mathrm{~mm} \times 0.82 \mathrm{~mm} \times 8.33 \mathrm{~mm}$.

Figure 9 shows the 3D distributions of particles within $30 \mathrm{~s}$ when fluorescein was injected into the chamber. Figure $9 \mathrm{a}, \mathrm{b}$ presents the initial diffusion process of the fluorescein solution aerosols in the chamber in the first $10 \mathrm{~s}$, and Figure 9a marks out the position of the inlet nozzle. It can be observed in Figure $9 a, b$ that the aerosols are spreading in the bottom and tend to rise up along the wall. As shown in Figure 9c,d, it can be observed that the density of aerosols at the bottom area becomes stronger, and aerosols keep rising from 10th to 20th second. Lastly, according to Figure 9e,f, the fluorescence signals get much stronger during the atomization process. The turbulence is clearly found in the upper area of the chamber. In the entire diffusion process, it took about 20-30 s for the chamber to be filled with fluorescein solution aerosols, which was quite slow. Therefore, the 3D imaging temporal resolution of $5 \mathrm{~s}$ could still have useful value.

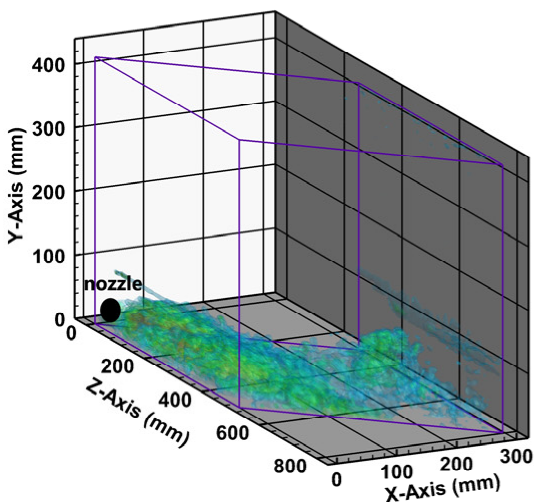

(a)

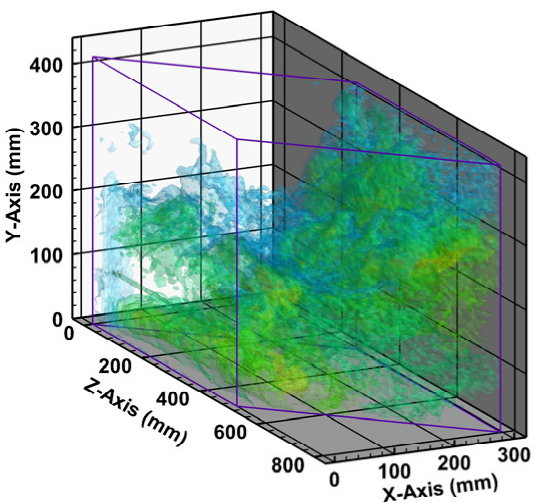

(d)

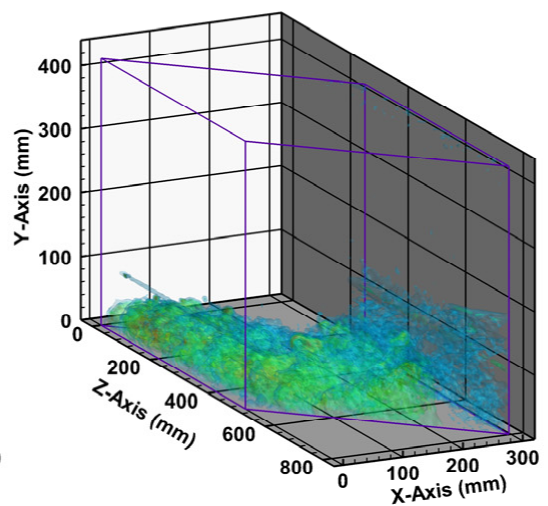

(b)

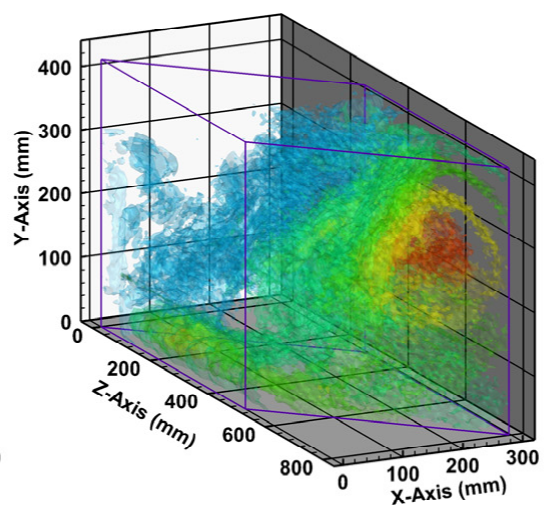

(e)

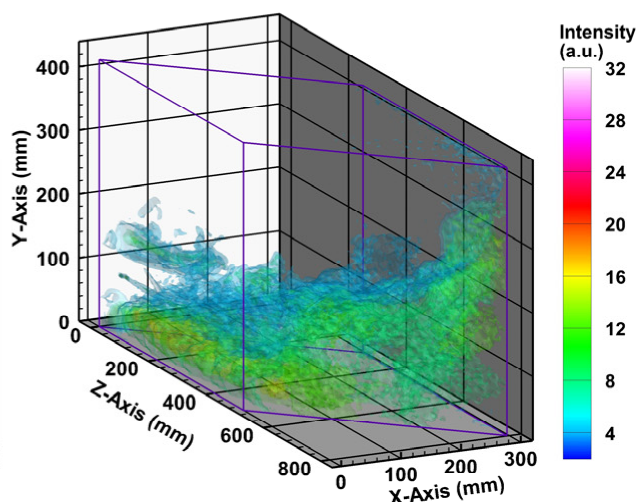

(c)

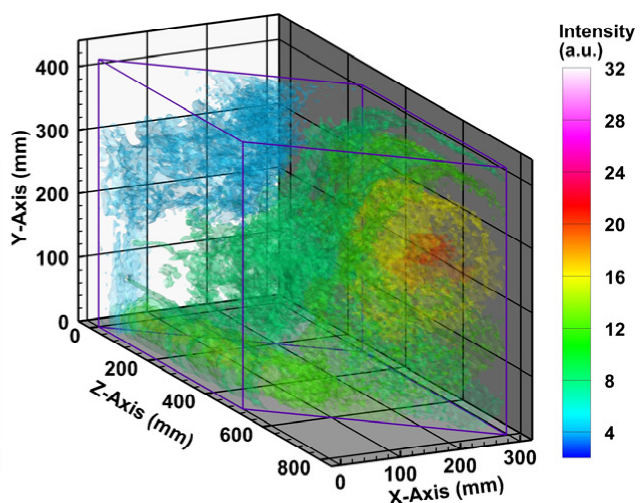

(f)

Figure 9. The 3D intensity distribution of PLIF images in 0-5 s (a), 5-10 s (b), 10-15 s (c), 15-20 s (d), 20-25 s (e) and 25-30 s (f). The concentration of the atomized fluorescein solution is $0.1 \mathrm{~g} / \mathrm{L}$, and the moving speed of the sliding platform is $10 \mathrm{~cm} / \mathrm{s}$. The signals were obtained with the laser power of $100 \mathrm{~mW}$, a $25 \mathrm{~ms}$ frame, and an atomization rate of $0.5 \mathrm{~mL} / \mathrm{s}$.

If more complicated data processing methods such as interpolation and curve fitting are applied for this system, it's feasible to demonstrate the animation effects of the variation 
of 3D distribution, which can promote further studies on the dynamics of biological aerosols.

\section{Conclusions}

In this paper, a 3D modeling system was designed and demonstrated to visualize the distribution of relative concentrations of fluorescein particles using the PLIF technique. The laser plane at an angle of $60^{\circ}$ was utilized as the excitation source to induce fluorescence, and a sCMOS camera was employed to collect PLIF images. The background subtraction, pixel merging, and wavelet denoising methods were selected for image processing. In the experiments, the system was used to image $2 \mathrm{D}$ and $3 \mathrm{D}$ distributions of particles, and then the concentration variation of fluorescein in the atomization process was analyzed to verify the reliability and performance of the system. Despite a relatively lower temporal and spatial resolution of the system, the experiment results prove that the system is able to visualize the $3 \mathrm{D}$ diffusion process of aerosols in a $500 \mathrm{~mm} \times 500 \mathrm{~mm} \times 1000 \mathrm{~mm}$ chamber, which is important for modeling and studying the distribution, leakage, and diffusion of bioaerosols. According to fluorescence spectra of bioaerosol particles, the system can select the specific fluorescence signals for imaging and analysis. Furthermore, by placing simulation models in the chamber, the system can be used to analyze a more realistic diffusion process of particles in various confined environments in future studies.

Author Contributions: Conceptualization, S.C. and Y.Z.; methodology, P.G.; software, Y.C.; validation, Y.C. and P.G.; investigation, S.C. and Y.C.; data curation, Y.C., H.C., and H.W.; writing-original draft preparation, S.C., H.C., and H.W.; writing-review and editing, S.C., H.C., and H.W.; supervision, Y.Z. All authors have read and agreed to the published version of the manuscript.

Funding: This work is supported by the National Natural Science Foundation of China (No.42005137) and the Fundamental Research Funds for the Central Universities (No.2020CX02002).

Institutional Review Board Statement: Not applicable.

Informed Consent Statement: Not applicable.

Data Availability Statement: Not applicable.

Conflicts of Interest: The authors declare no conflict of interest.

\section{References}

1. Kim, K.H.; Kabir, E.; Jahan, S.A. Airborne bioaerosols and their impact on human health. J. Environ. Sci. 2018, 67, 23-35. [CrossRef]

2. Fröhlich-Nowoisky, J.; Kampf, C.J.; Weber, B.; Huffman, J.A.; Pöhlker, C.; Andreae, M.O.; Pöschl, U. Bioaerosols in the Earth system: Climate, health, and ecosystem interactions. Atmos. Res. 2016, 182, 346-376. [CrossRef]

3. Fiegel, J.; Clarke, R.; Edwards, D.A. Airborne infectious disease and the suppression of pulmonary bioaerosols. Drug Discov. Today. 2006, 11, 51-57. [CrossRef]

4. Fung, F.; Hughson, W.G. Health effects of indoor fungal bioaerosol exposure. Appl. Occup. Environ. Hyg. 2003, 18, 535-544. [CrossRef] [PubMed]

5. Heo, K.J.; Lim, C.E.; Kim, H.B.; Lee, B.U. Effects of human activities on concentrations of culturable bioaerosols in indoor air environments. J. Aerosol Sci. 2017, 104, 58-65. [CrossRef]

6. Holler, S.; Chang, R.K.; Hill, S.C.; Pinnick, R.G.; Niles, S.; Bottiger, J.R. Single-shot fluorescence spectra of individual micrometersized bioaerosols illuminated by a 351- or a 266-nm ultraviolet laser. Opt. Lett. 1999, 24, 116-118.

7. Pan, Y.L.; Hartings, J.; Pinnick, R.G.; Hill, S.C.; Halverson, J.; Chang, R.K. Single-particle fluorescence spectrometer for ambient aerosols. Aerosol Sci. Tech. 2003, 37, 628-639. [CrossRef]

8. Kiselev, D.; Bonacina, L.; Wolf, J.P. Individual bioaerosol particle discrimination by multi-photon excited fluorescence. Opt. Express 2011, 19, 24516-24521. [CrossRef] [PubMed]

9. Joshi, D.; Kumar, D.; Maini, A.K.; Sharma, R.C. Detection of biological warfare agents using ultra violet-laser induced fluorescence LIDAR. Spectrochim. Acta A. 2013, 112, 446-456. [CrossRef]

10. Gottfried, J.L.; De Lucia, F.C.; Munson, C.A.; Miziolek, A.W. Standoff detection of chemical and biological threats using laser-induced breakdown spectroscopy. Appl. Spectrosc. 2008, 62, 353-363. [CrossRef]

11. Sharma, R.C.; Kumar, D.; Kumar, S.; Joshi, D.; Srivastva, H.B. Standoff detection of biomolecules by ultraviolet laser-induced fluorescence LIDAR. IEEE Sens. J. 2015, 15, 3349-3352. [CrossRef] 
12. Kim, M.S.; Lefcourt, A.M.; Chen, Y.R. Multispectral laser-induced fluorescence imaging system for large biological samples. Appl. Optics 2003, 42, 3927-3934. [CrossRef] [PubMed]

13. Cho, K.Y.; Satija, A.; Pourpoint, T.L.; Son, S.F.; Lucht, R.P. High-repetition-rate three-dimensional OH imaging using scanned planar laser-induced fluorescence system for multiphase combustion. Appl. Optics 2014, 53, 316-326. [CrossRef] [PubMed]

14. Miller, V.A.; Troutman, V.A.; Hanson, R.K. Near-kHz 3D tracer-based LIF imaging of a co-flow jet using toluene. Mea. Sci. Technol. 2014, 25, 075403. [CrossRef]

15. Cai, W.; Li, X.; Ma, L. Practical aspects of implementing three-dimensional tomography inversion for volumetric flame imaging. Appl. Optics 2013, 52, 8106-8116. [CrossRef] [PubMed]

16. Ma, L.; Lei, Q.; Capil, T.; Hammack, S.D.; Carter, C.D. Direct comparison of two-dimensional and three-dimensional laser-induced fluorescence measurements on highly turbulent flames. Opt. Lett. 2017, 42, 267-270. [CrossRef]

17. Halls, B.R.; Thul, D.J.; Michaelis, D.; Roy, S.; Meyer, T.R.; Gord, J.R. Single-shot, volumetrically illuminated, three-dimensional, tomographic laser-induced-fluorescence imaging in a gaseous free jet. Opt. Express 2016, 24, 10040-10049. [CrossRef]

18. Li, T.; Pareja, J.; Fuest, F.; Schütte, M.; Zhou, Y.; Dreizler, A.; Böhm, B. Tomographic imaging of OH laser-induced fluorescence in laminar and turbulent jet flames. Mea. Sci. Technol. 2017, 29, 015206. [CrossRef]

19. Farsund, Ø.; Rustad, G.; Kasen, I.; Haavardsholm, T.V. Required spectral resolution for bioaerosol detection algorithms using standoff laser-induced fluorescence measurements. IEEE Sens. J. 2010, 10, 655-661. [CrossRef]

20. Skiba, A.W.; Wabel, T.M.; Carter, C.D.; Hammack, S.D.; Temme, J.E.; Lee, T.; Driscoll, J.F. Reaction layer visualization: A comparison of two PLIF techniques and advantages of kHz-imaging. Proc. Combust. Inst. 2017, 36, 4593-4601. [CrossRef]

21. Wu, Y.; Xu, W.; Lei, Q.; Ma, L. Single-shot volumetric laser induced fluorescence (VLIF) measurements in turbulent flows seeded with iodine. Opt. Express 2015, 23, 33408-33418. [CrossRef]

22. Blomberg, S.; Zhou, J.; Gustafson, J.; Zetterberg, J.; Lundgren, E. 2D and 3D imaging of the gas phase close to an operating model catalyst by planar laser induced fluorescence. J. Phys. Condens. Matter 2016, 28, 453002. [CrossRef] [PubMed]

23. Wellander, R.; Richter, M.; Aldén, M. Time-resolved (kHz) 3D imaging of OH PLIF in a flame. Exp. Fluids 2014, 55, 1-12. [CrossRef]

24. Pareja, J.; Johchi, A.; Li, T.; Dreizler, A.; Böhm, B. A study of the spatial and temporal evolution of auto-ignition kernels using time-resolved tomographic OH-LIF. Proc. Combust. Inst. 2019, 37, 1321-1328. [CrossRef]

25. Sjöback, R.; Nygren, J.; Kubista, M. Absorption and fluorescence properties of fluorescein. Spectrochim. Acta A. 1995, 51, L7-L21. [CrossRef]

26. Han, M.; Liu, Y.; Xi, J.; Guo, W. Noise smoothing for nonlinear time series using wavelet soft threshold. IEEE Signal Process. Lett. 2006, 14, 62-65. [CrossRef]

27. Valencia, D.; Orejuela, D.; Salazar, J.; Valencia, J. Comparison analysis between rigrsure, sqtwolog, heursure and minimaxi techniques using hard and soft thresholding methods. In Proceedings of the Symposium on Signal Processing, Images and Artificial Vision (STSIVA), Bucaramanga, Colombia, 31 August-16 September 2016.

28. Chen, S.; Yu, Y.L.; Wang, J.H. Inner filter effect-based fluorescent sensing systems: A review. Anal. Chim. Acta. 2018, 999, 13-26. [CrossRef]

29. Wind, L.; Szymanski, W.W. Quantification of scattering corrections to the Beer-Lambert law for transmittance measurements in turbid media. Mea. Sci. Technol. 2002, 13, 270. [CrossRef] 